\title{
Análisis gravitrópico de la raíz primordial de plantas mesoamericanas, en condiciones de microgravedad simulada
}

\author{
Brenda Medina ${ }^{1}$ \\ Cesar Rodríguez ${ }^{2}$
}

\section{RESUMEN}

Esta investigación muestra datos del crecimiento y curvatura gravitrópica de seis especies de importancia actual para el área Mesoamericana, Paseolus vulgaris var. Criolla, Paseolus vulgaris var. Amadeus 77, Phaseolus lunatus Phaseolus acutifolious, Vigna unguiculata y Sorghum bicolor, bajo condiciones de microgravedad simulada en clinostato de un eje, así como cortes histológicos de los tejidos de las muestras clinorotadas y las muestras Control $1 \mathrm{~g}$.

Tiene como objetivo comprender el comportamiento de la raíz primordial de plantas actualmente cultivadas en Mesoamérica, cuando la fuerza neta sobre la vertical es menor que la fuerza gravitacional; Esto con la intención de identificar las especies vegetales que pudieran tener un crecimiento óptimo a baja gravedad.

Las pruebas de laboratorio se llevaron a cabo en las instalaciones de la Facultad de Ciencias Espaciales UNAH. Sembrando en discos de Petri con un sustrato llamado agar y dentro de cámaras húmedas para mantener iguales condiciones de humedad y temperatura. Luego de germinadas las raíces se procedió al proceso de clinorotación de la muestra llamada "Clinorotada" (sometida a microgravedad) durante sesiones de dos horas; tomando fotografías cada treinta minutos para su posterior análisis de comparación. Los datos fueron analizados mediante el programa ImageJ, y tabulados mediante Excel.

Phaseolus lunatus, Vigna unguiculata y Soghum bicolor son tres especies en las que se pudo observar que su crecimiento no ha sido considerablemente afectado a baja gravedad. Por lo que son especies que podrían ser consideradas para mayores estudios en microgravedad a bordo de sondas espaciales.

\footnotetext{
${ }^{1}$ Maestría Académica Regional Centroamericana en Astronomía y Astrofísica, Depto. Astronomía y Astrofísica, Facultad de Ciencias Espaciales, UNAH. brendamedinapaz@gmail.com

${ }^{2}$ Asesor metodológico de la Maestría Académica Regional Centroamericana en Astronomía y Astrofísica, Depto. Astronomía y Astrofísica, Facultad de Ciencias Espaciales, UNAH. cesaronca@gmail.com
} 
Palabras clave: Arquitectura, sostenibilidad, vivienda básica, Honduras

\section{ABSTRACT}

This research shows data of the growth and gravitropic curvature of six species of current importance for the Mesoamerican area, Paseolus vulgaris var. Creole, Paseolus vulgaris var. Amadeus 77, Phaseolus lunatus Phaseolus acutifolious, Vigna unguiculata and Sorghum bicolor; Under conditions of microgravity simulated in clinostat of an axis, as well as histological cuts of the tissues of the Clinorotated samples and the Control $1 \mathrm{~g}$ samples.

It aims to understand the behavior of the primordial root of plants currently cultivated in Mesoamerica, when the net force on the vertical is smaller than the gravitational force; this was done with the intention of identifying the plant species that could have optimal growth at low gravity.

The laboratory tests were carried out at the facilities of the Faculty of Space Sciences UNAH. Sowing in Petri dishes with a substrate called agar and inside humid chambers to maintain equal humidity and temperature conditions. After the roots were germinated we proceeded to the process of clinorotation of the sample called "Clinorotated" (subjected to microgravity) during sessions of two hours; Taking photographs every thirty minutes for further comparison analysis. The data were analyzed using the ImageJ program, and tabulated using Excel.

Phaseolus lunatus, Vigna unguiculata and Soghum bicolor are three species in which it was observed that their growth has not been significantly affected to low gravity. So they are species that could be considered for further studies in microgravity onboard space probes.

Keywords: microgravity, clinostat, growth under microgravity, gravitropic curvature. 


\section{INTRODUCCIÓN}

Los estudios en microgravedad son realizados para analizar el desarrollo de entes biológicos, y comprender como estos se comportan en nuevos ambientes. Hoy en día, investigaciones han desarrollado plataformas experimentales en el estudio de la influencia de la gravedad alterada. En consecuencia, nuestro conocimiento de los efectos de la gravedad y la microgravedad ha aumentado en gran medida. Hallazgos clave de la investigación en biología gravitacional cubren todos los niveles de la biología, que van desde las proteínas aisladas, las células individuales y tejidos de organismos complejos.

Esta investigación propone aportar conocimiento científico a la rama de la Astrobiología, especialmente la Biología Gravitacional; fundamentar las bases teóricas mediante experimentación y análisis de cultivos simulando la microgravedad en tierra, de lo que pronto será el cultivo espacial, practicado en las sondas al realizar largos viajes espaciales.

La comunidad científica astronómica y astrofísica en todas sus corrientes y derivaciones apunta en ese futuro inevitable de la humanidad a conquistar el espacio en comunidades autosustentables en otros planetas, satélites o cuerpos celestes en donde la vida podría desarrollarse. Es por ello que experimentos de microgravedad en organismos pluricelulares son simulados en todo el mundo, siendo ahora una carrera de naciones. Hasta el momento experimentos con especies actualmente cultivadas en mesoamerica no se han llevado a cabo en Honduras, por lo que se pretende ser pioneros del campo en el país y Centroamérica.

Ésta investigación consistió en un análisis del ángulo de la curvatura gravitrópica (curvatura en función de la gravedad) y crecimiento longitudinal de la raíz primordial de especies nativas mesoamericanas, en condiciones de microgravedad simulada en un clinostato de un solo eje.

Se entiende como microgravedad a la millonésima parte de la gravedad terrestre expresada como " $\mu g$ " o micro-g, y oscila entre rangos de 10-3 a 10-6; que en una nave espacial es formada por el llamado "nerviosismo gravitatorio" vibraciones causadas por la maquinaria a bordo, movimiento de los astronautas, operaciones en el motor y más (Herranz Raúl, et al, 2013). 


\section{Percepción de la gravedad}

Para los organismos sésiles (que no se desplazan) saber dónde es arriba y abajo es transcendental, ya que de esta manera son capaces de ordenar a sus células crecer las raíces hacia el fondo de la tierra y los brotes fuera, permitiendo así la correcta absorción de nutrientes y la captación de energía solar para la realización de sus funciones.

Para lograr establecer una relación de arriba y abajo las plantas poseen células especializadas (Estatocitos) capaces de percibir la fuerza gravitatoria ejercida por la Tierra sobre todos los cuerpos (gravipercepción). Dentro de estas células se encuentran amiloplastos (vesículas celulares que contienen almidón) llamados estatolitos, los cuales se sedimentan en las células siguiendo el vector de gravedad.

Esta sedimentación ayudada por la actina (Proteína globular que conforma los Microfimalentos, unos de los tres componentes del citoesqueleto celular) permite que un gravireceptor (canales mecano-sensitivos o acarreadores de flujo de salida de auxina) en la membrana plasmática, transforme el estímulo físico en una señal fisiológica.

Figura 1. Modelo de trabajo para los mecanismos de retroalimentación de crecimiento punta gravitrópica.

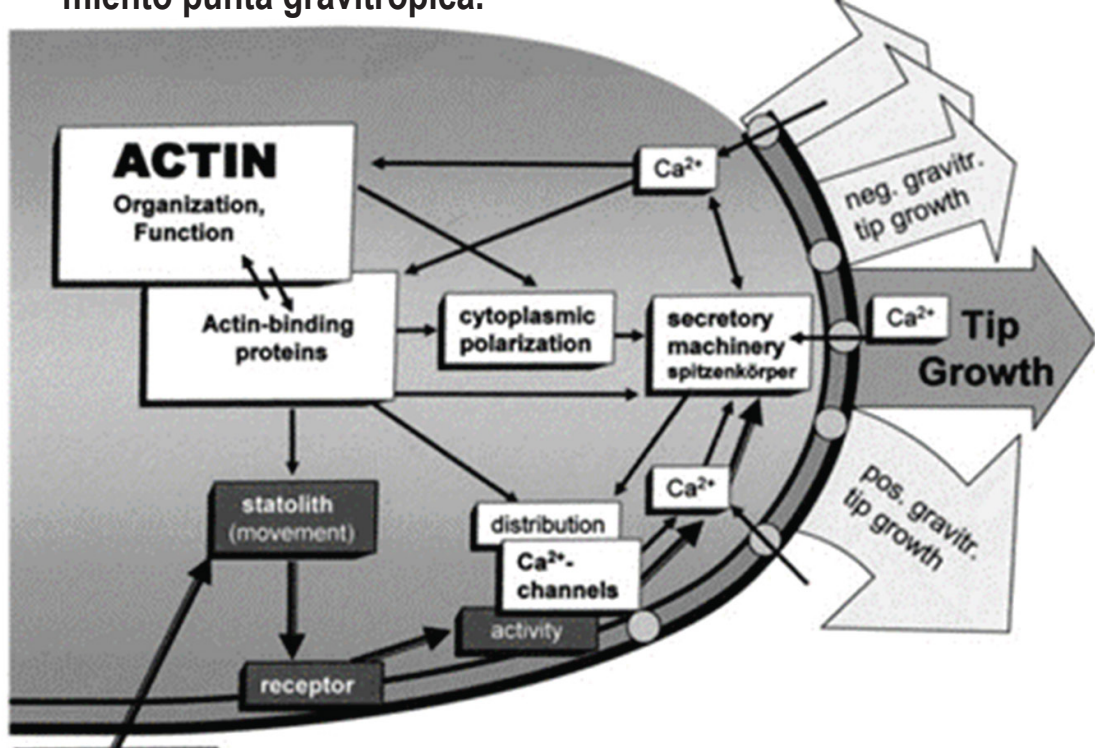

\section{GRAVITY}

Fuente: Braun \& Limbach.2006. Rhizoids and protonemata of characean algae model cells for research on polarized growth and plant gravity sensing [Artículo Cientifico] 
Esta señal inicia una cascada de eventos que envuelve un cambio local de la actividad de los canales de calcio. Un incremento de la concentración de Ca+2 citoplasmático libre en la célula inferior conduce a la diferencia de crecimiento de células sub-apicales (Células encargadas de formar el tejido diferenciado en los vegetales) opuestas en forma de una suave inclinación hacia abajo como se muestra en la figura 1 (Braun \& Limbach, 2006).

\section{Simulación de microgravedad}

La Microgravedad en tierra puede ser simulada mediante varios métodos entre ellos, Clinostatos (1D, 2D, 3D), máquinas de posicionamiento aleatorio, y levitación diamagnética (Herranz Raúl, et al, 2013).

Para efecto de este estudio se trabajó con un Clinostato de un solo eje (1D), es un dispositivo experimental que puede contrarrestar el vector de gravedad alrededor de uno 0 dos ejes rotacionales según sea el caso; este es un fenómeno de reacción lenta.

\section{METODOLOGÍA}

La investigación de enfoque cuantitativo, contó con un diseño cuasi-experimental, de alcance explicativo y una temporalidad transversal. La población la constituyen plantas importantes para la agricultura y el consumo humano cultivadas en la región mesoamericana. Esta investigación se llevó a cabo en la Facultad de Ciencias Espaciales de la Universidad Nacional Autónoma de Honduras, Ciudad Universitaria, Tegucigalpa.

La muestra no es representativa de toda la población ya que debe cumplir características importantes de experimentación. Dentro de ellas, es que sean de pequeño tamaño para poder sembrarse en el disco de Petri y este pueda fijarse al clinostato. $Y$ además sean de rápida germinación para aprovechar al máximo el tiempo destinado a la experimentación.

Dentro de las unidades de análisis encontramos, Phaseolus vulgaris (frijol común) var. SEDA criolla y var. Amadeus 77, Phaseolus acutifolius (Frijol Tepary) var. 11F-3870, Phaseolus lunatus (Frijol lima), Sorghum bicolor (Sorgo) variedad Sureño, Vignia unguiculata (Caupí o Cawpea) var. Gorda. 


\section{Recolección de datos}

\section{Etapa de preparación}

Este proceso consiste en preparar cuatro capsulas de Petri, para el proceso de experimentación. Las muestras se nombraron como: "Control 1g", "Girada 90”", "Clinorotada", y copia de "Seguridad". Primeramente, se deben marcar las capsulas de Petri con una línea de referencia, esta nos permitió ubicar la dirección de colocación sobre un soporte de madera, siguiendo del vector de gravedad, y que estas pudieran germinar bajo gravedad natural.

\section{Figura 2. Preparación del agar.}

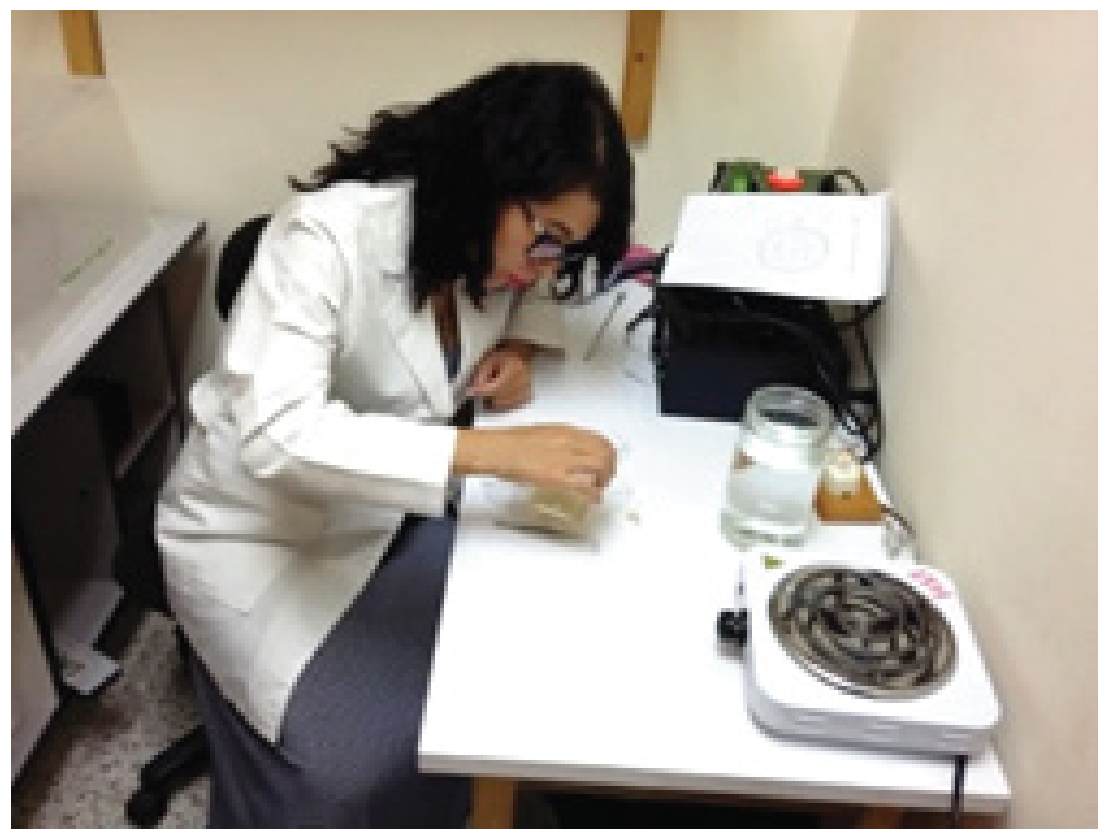

Fuente: Medina, Brenda.2017. Análisis gravitrópico de la raíz primordial de plantas mesoamericanas, en condiciones de microgravedad simulada.

Las semillas deben ser sembradas en un sustrato especial que nos permita observar el desarrollo de las plántulas, es por ello que se realizó en Agar; este fue preparado al $1.5 \%$ (esto es $1.5 \mathrm{~g}$ de agar en $100 \mathrm{ml}$ de agua). El agar se prepara calentando el disolvente $\left(80^{\circ} \mathrm{C}\right.$ aproximadamente) y mezclarlo con agar en polvo hasta obtener un tono claro casi transparente tan como se muestra en la Figura 2. Se debe esperar a que se enfrié (entre 50 y $45^{\circ} \mathrm{C}$ ) antes de trasvasar a los discos de Petri y dejar enfriar 
aún más, pero no dejar a que la mezcla se solidifique por completo, ya que de suceder las semillas no podrán ser plantadas. En cada capsula se deben sembrar de 6 a 9 semillas (o la cantidad según el tamaño de las semillas) de la misma especie asegurándose de que estén sumergidas a la mitad para asegurar la captación de oxigeno; luego el disco se coloca en dirección vertical (en un soporte de madera) siguiendo el vector de gravedad, finalmente fueron colocadas en una cámara húmeda (entre $60 \%$ - 100\%) para la germinación; el proceso de experimentación puede comenzarse cuando la raíz ha crecido y alcanzado entre $5 \mathrm{~mm}$ a $10 \mathrm{~mm}$ de longitud.

\section{Etapa de experimentación}

Esta etapa consiste en observar la influencia de la clinorotación en el gravitropísmo y crecimiento de las raíces. Comenzó cuando la muestra "Girada $90^{\circ}$ " se giró en $90^{\circ}$ en cualquier dirección (derecha o izquierda), se marcó una nueva línea en distinto color (rojo) que muestra la nueva dirección que sigue el vector de gravedad. Ubicada en la nueva posición se fotografió para su posterior análisis.

Se colocó la muestra "Clinorotada" en el centro del clinostato con cinta adhesiva doble cara asegurándose que se encontrará en dirección vertical hasta que la clinorotación comenzará. Se fotografiaron las 3 muestras ("Girada 90", "Clinorotada" y "Control 1g") siguiendo algunos parámetros (Figura 3). El fondo debe ser oscuro y homogéneo, con suficiente iluminación artificial ubicada arriba de las muestras, y una cámara de buena resolución. Seguidamente se procedió a la clinorotación a la velocidad deseada (70rpm y $90 \mathrm{rpm}$ ) en sesiones de 2 horas.

Se tomaron fotografías de los tres discos de Petri cada 30 minutos. Tratando de parar el clinostato el menor tiempo posible. Registrando el final del tiempo de observación Concluida la clinorotación se procede a realizar los cortes histológicos. Se coloca la raíz en un portaobjetos y se hacen pequeños cortes longitudinales o transversales con navaja (Gillette), se colorea la muestra con Toluidina y se espera el tiempo prudente para la reacción. Se cubre con un cubreobjetos y se hace el respectivo estudio con un microscopio. Finalmente se toma una fotografía con una cámara para microscopio, para su posterior análisis. 
Figura 3. Toma de fotografías durante la clinorotación.

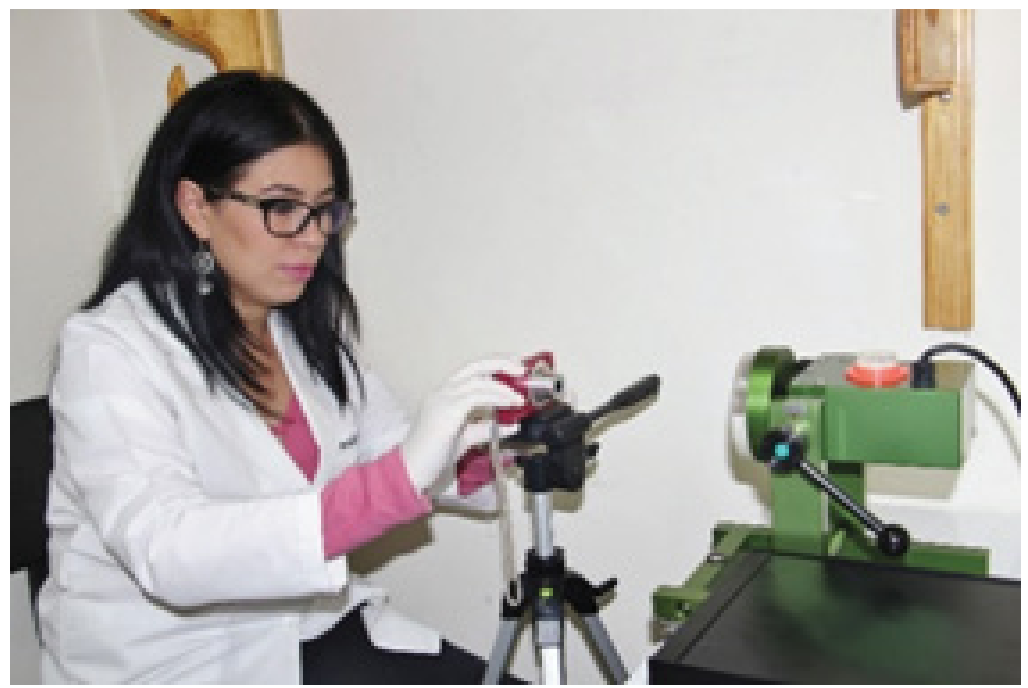

Fuente: Medina, Brenda.2017. Análisis gravitrópico de la raíz primordial de plantas mesoamericanas, en condiciones de microgravedad simulada.

\section{Plan de análisis}

\section{Análisis: Curvatura de la raíz}

Para el análisis de la curvatura de la raíz, se utilizó un programa de procesamiento de imágenes llamado "ImageJ" que permite medir ángulos de las curvaturas de las raíces. Se miden los ángulos de curvatura de las muestras "Girada $90^{\circ}$ " y "Clinorotada" obteniendo el ángulo de curvatura real, restando el obtenido de $180^{\circ}$ esto es $\left(180^{\circ}\right.$ - obtenido).

Luego de medir todos los ángulos de curvatura de ambas muestras ("Girada 90" y "Clinorotada") para cada tiempo, se calculó el valor promedio. Se hizo la respectiva comparación entre ambas muestras. Finalmente se calculó la tasa angular promedio de la raíz en grados por hora.

\section{Análisis: Tasa de crecimiento}

Para el análisis de la tasa de crecimiento se utilizó las imágenes de la muestra "Control" y la muestra "Clinorotada" con el propósito de analizar la diferencia entre ambos casos. Ya que la muestra "Control" es continuamente estimulada por la gravedad terrestre, haciendo crecer las raíces en esa dirección. Mientras que la muestra "Clino- 
rotada" no es afectada por fuerza de gravedad impulsándola en alguna dirección en específica (esta se encuentra en microgravedad durante las dos horas de experimentación).

La medición del crecimiento se ejecutó mediante el programa "ImageJ" en donde se debe configurar una escala apropiada, colocando una regla a la misma profundidad de las raíces, antes de la toma de fotografías o dibujar en el disco de Petri una línea de un centímetro, que también es válida; así sabremos en escala de centímetros cuanto han crecido las raíces. Se registró la medida de cada semilla de ambas muestras y se obtuvo un promedio para la muestra "Control" y uno para la muestra "Clinorotada" para finalmente calcular la tasa de crecimiento promedio en $\mathrm{cm} / \mathrm{h}$. Así como un análisis estructural de los cortes histológicos (Figura 4) para ultimar las conclusiones al respecto.

Figura 4. Análisis de las fotografías de los cortes histológicos. Medina, Brenda.2017. Análisis gravitrópico de la raíz primordial de plantas mesoamericanas, en condiciones de microgravedad simulada.

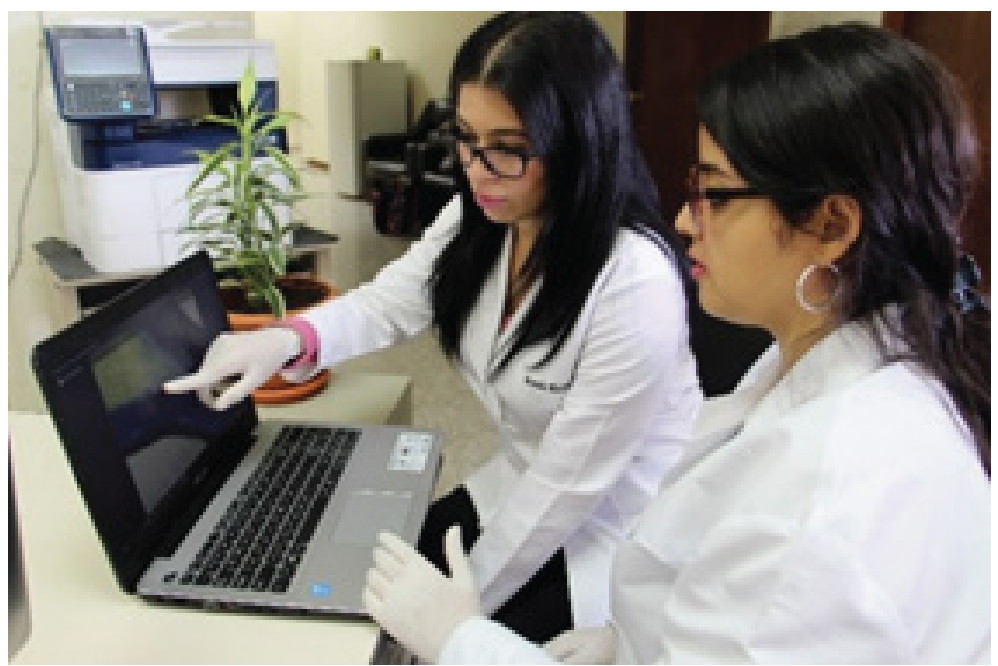

Fuente: Medina, Brenda.2017. Análisis gravitrópico de la raíz primordial de plantas mesoamericanas, en condiciones de microgravedad simulada.

\section{RESULTADOS}

En el proceso de experimentación se realizaron tres pruebas, cada una involucró las 6 especies en investigación. La primera y última prueba experimental que se desarro- 
lló, la muestra se sometió a una velocidad de rotación de 70 revoluciones por minuto. En donde esta contrarrestó la fuerza gravitacional, logrando una aceleración que actúa sobre las especies durante la clinorotación de $8.22 \times 10-2 \mathrm{~m} / \mathrm{s} 2$. Mientras que la última se realizó a velocidad de $90 \mathrm{rpm}$ logrando una aceleración de 1.359x10-1 $\mathrm{m} / \mathrm{s} 2$. Las cuales fueron calculadas mediante la formula

$$
\frac{F c}{\mathrm{~g}}=\frac{(\pi / 30)^{2} r}{r} \frac{\mathrm{w}^{2}}{9.81}=1.12 \times 10^{-3} r w^{2}
$$

\section{Paseolus vulgaris var. Criolla (70 rpm)}

Gráfico 1. Arriba, tasa angular promedio; La muestra "Girada $90^{\circ}$ " presentó una tasa angular promedio (Curvatura gravitrópica) de $24.080^{\circ}$ en una hora, mientras que la muestra Clinorotada fue de $4.321^{\circ}$ por hora. Abajo, Tasa de crecimiento promedio; muestra Control $1 \mathrm{~g}$ creció 0.130 $\mathrm{cm}$ en una hora, mientras que la muestra Clinorotada creció $0.212 \mathrm{~cm}$ en la misma hora.

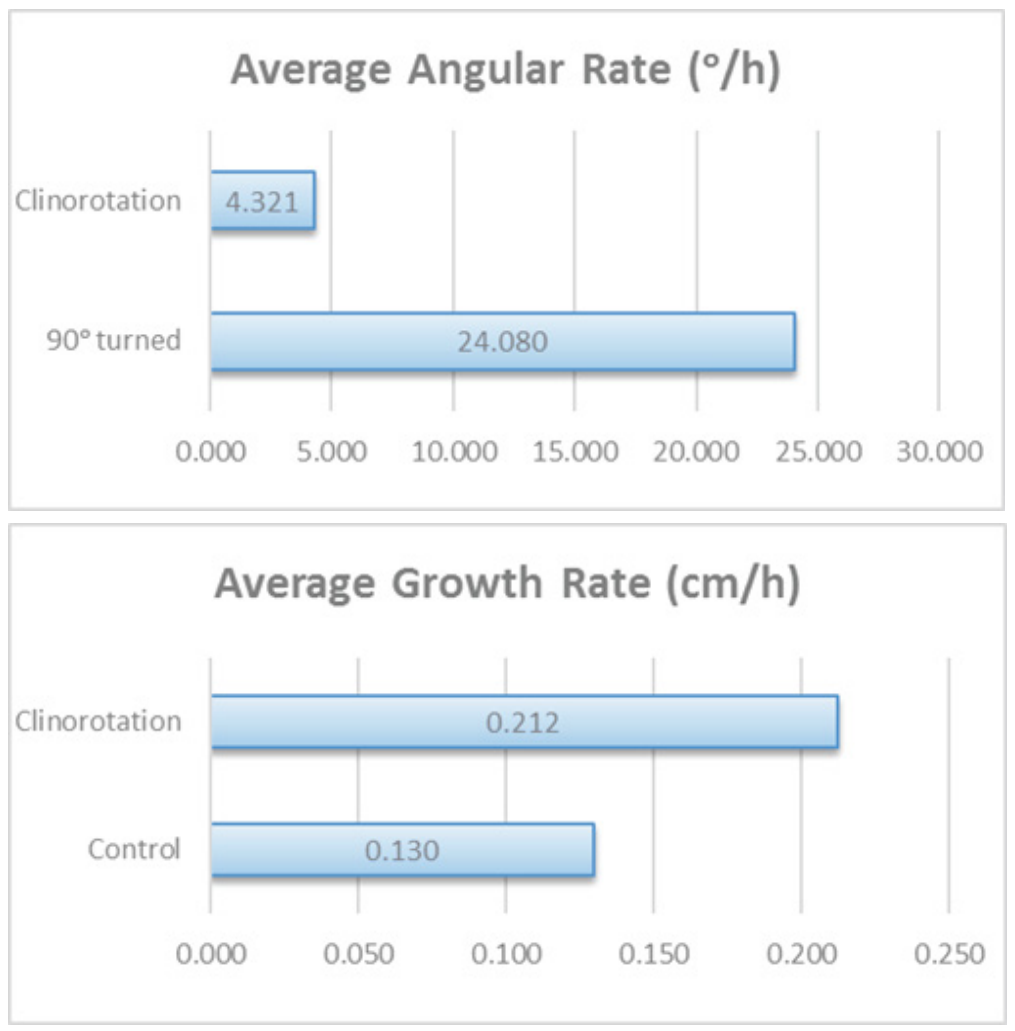

Fuente: Medina, Brenda.2017. Análisis gravitrópico de la raíz primordial de plantas mesoamericanas, en condiciones de microgravedad simulada 
Gráfico 2. Muestra la longitud de crecimiento en centímetros vrs. El tiempo de germinación, para Phaseolus vulgaris var. Criolla. Muestra Control $1 \mathrm{~g}$ (Izquierda) y la muestra Clinorotada (Derecha).

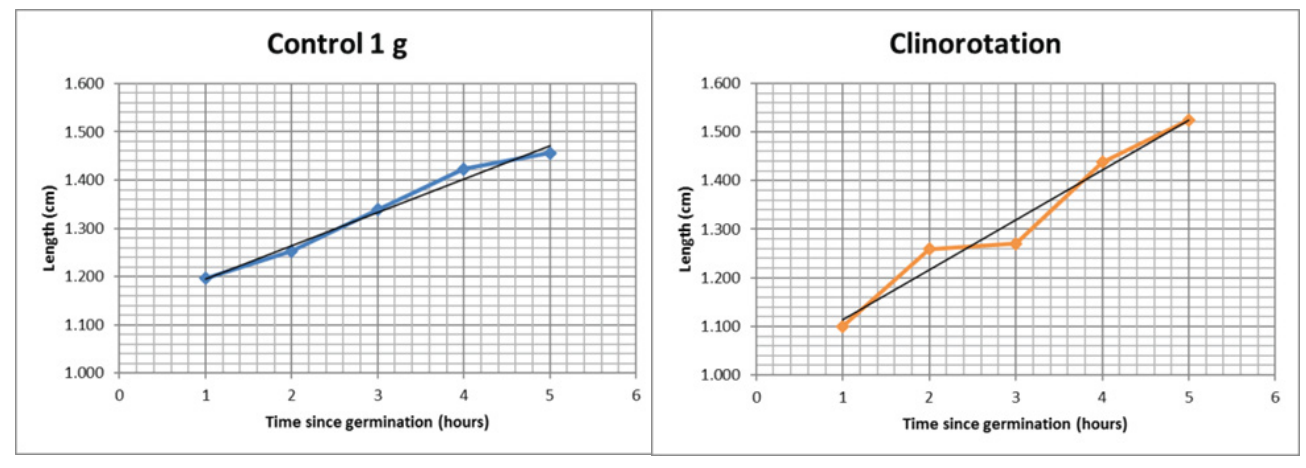

Fuente: Medina, Brenda.2017. Análisis gravitrópico de la raíz primordial de plantas mesoamericanas, en condiciones de microgravedad simulada

Corte Histológico Paseolus vulgaris var. Criolla (70 rpm)

Figura 5. Control 1g. (Corte longitudinal a 400 veces).

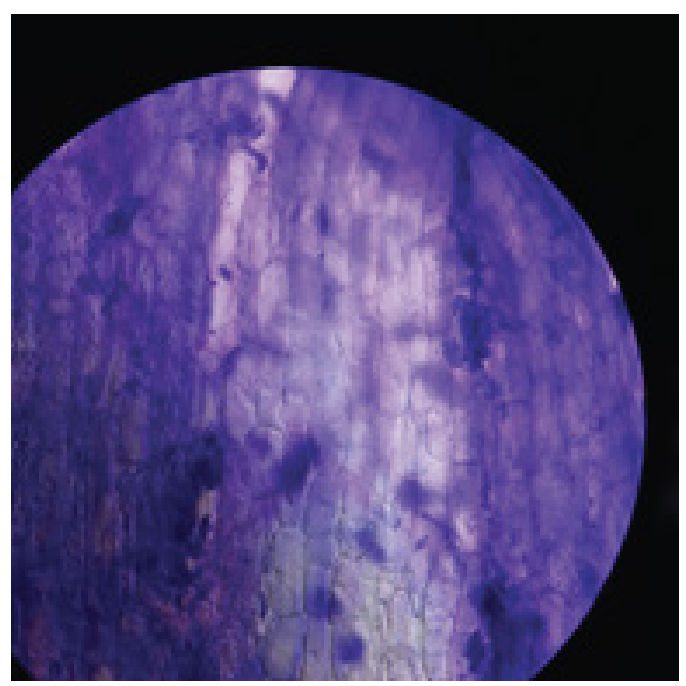

Fuente: Medina, Brenda.2017. Análisis gravitrópico de la raíz primordial de plantas mesoamericanas, en condiciones de microgravedad simulada 
Figura 6. Clinorotada (Corte longitudinal a 1000 veces).

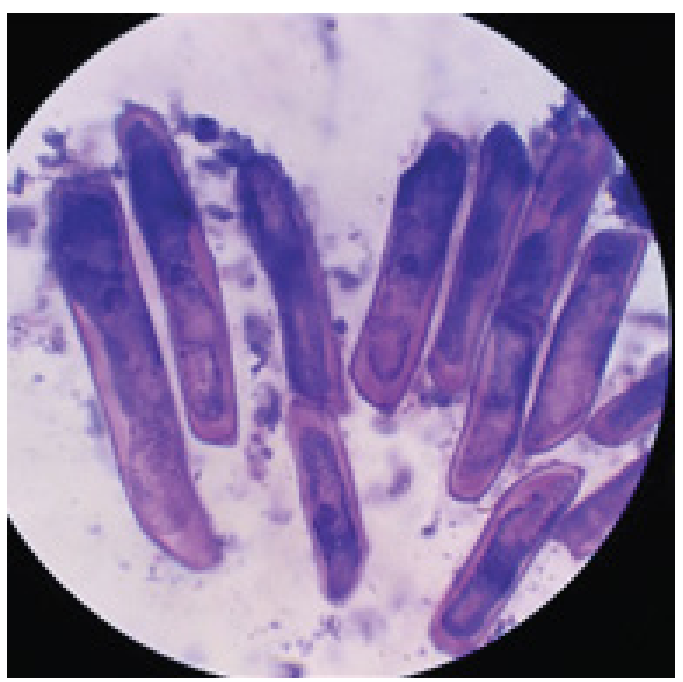

Fuente: Medina, Brenda.2017. Análisis gravitrópico de la raíz primordial de plantas mesoamericanas, en condiciones de microgravedad simulada

Phaseolus vulgaris var. mejorada "Amadeus 77" (70 rpm)

Gráfico 3. Arriba, tasa angular promedio; la muestra Girada $90^{\circ}$ mostro una curvatura de $8.827^{\circ}$ en una hora, y la muestra Clinorotada $9.012^{\circ}$. Abajo, tasa de crecimiento promedio; la muestra control $1 \mathrm{~g}$. creció $0.069 \mathrm{~cm}$. En una hora, mientras que la muestra Clinorotada creció un poco más, logrando $0.085 \mathrm{~cm}$ en el mismo tiempo.

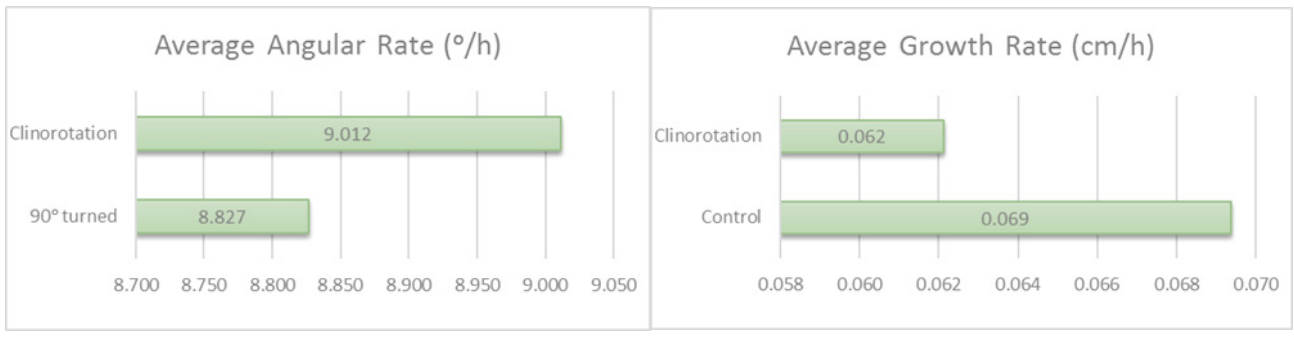

Fuente: Medina, Brenda.2017. Análisis gravitrópico de la raíz primordial de plantas mesoamericanas, en condiciones de microgravedad simulada 
Gráfico 4. Muestra la longitud de crecimiento en centímetros vrs. El tiempo de germinación, para Phaseolus vulgaris var. "Amadeus 77". Muestra Control 1g (Izquierda) y la muestra Clinorotada (Derecha).

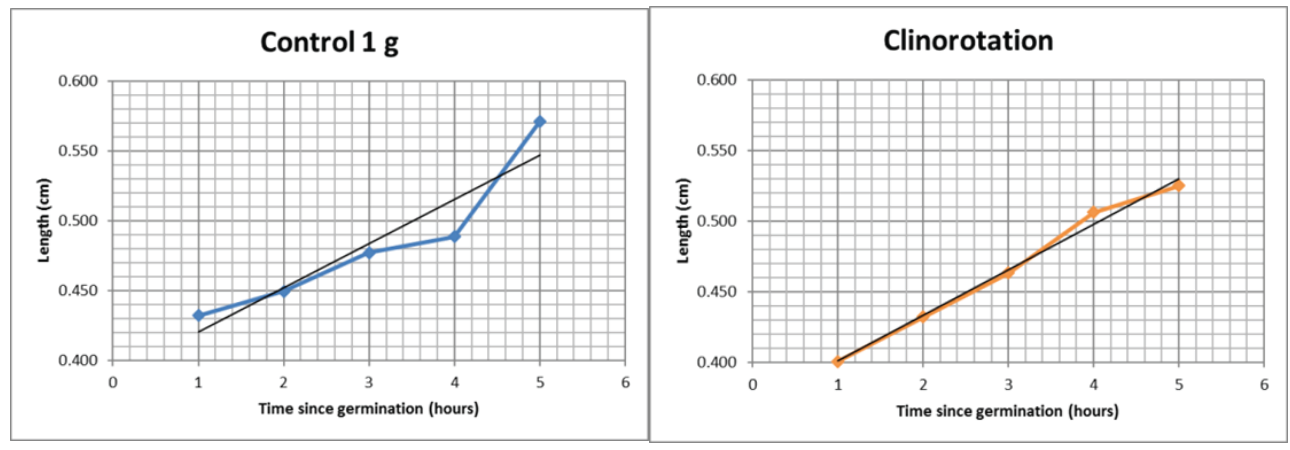

Fuente: Medina, Brenda.2017. Análisis gravitrópico de la raíz primordial de plantas mesoamericanas, en condiciones de microgravedad simulada

Corte Histológico Phaseolus vulgaris var. mejorada "Amadeus 77" (70 rpm)

Figura 7. Control 1g. (Corte longitudinal a 100 veces)

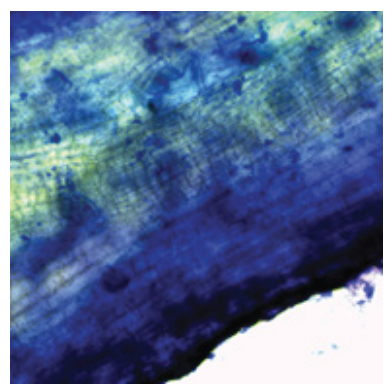

Fuente: Medina, Brenda.2017. Análisis gravitrópico de la raíz primordial de plantas mesoamericanas, en condiciones de microgravedad simulada

Figura 8. Clinorotada (Corte longitudinal a 100 veces).

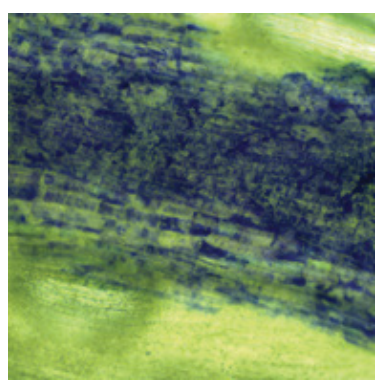

Fuente: Medina, Brenda.2017. Análisis gravitrópico de la raíz primordial de plantas mesoamericanas, en condiciones de microgravedad simulada 
Phaseolus acutifolious "Frijol Tepari" (90 rpm)

Gráfico 5. Izquierda, tasa angular promedio; La muestra Girada $90^{\circ}$ presento una tasa angular de $19.702 \%$, superando la muestra Clinoratada con $10.401 \% \mathrm{~h}$. Derecha, tasa de crecimiento promedio; ambas muestras estuvieron muy cercanas. La muestra Control $1 \mathrm{~g}$. creció $0.146 \mathrm{~cm}$ y la muestra Clinorotada creció $0.141 \mathrm{~cm}$, en un periodo de 1 hora.

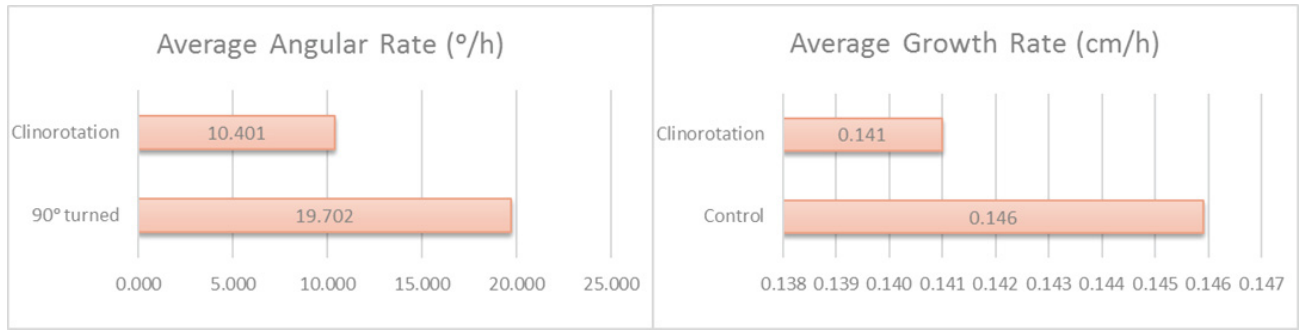

Fuente: Medina, Brenda.2017. Análisis gravitrópico de la raíz primordial de plantas mesoamericanas, en condiciones de microgravedad simulada

Gráfico 6. Muestra la longitud de crecimiento en centímetros vrs. El tiempo de germinación, para Phaseolus acutifolious. Muestra Control $1 \mathrm{~g}$ (Izquierda) y la muestra Clinorotada (Derecha).
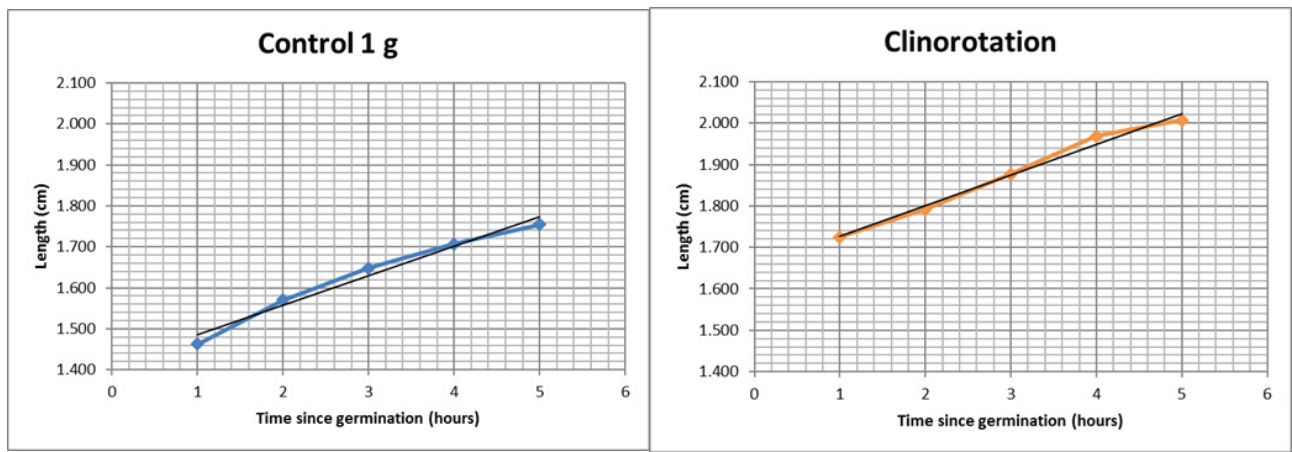

Fuente: Medina, Brenda.2017. Análisis gravitrópico de la raíz primordial de plantas mesoamericanas, en condiciones de microgravedad simulada

\section{Corte Histológico Phaseolus acutifolious (90 rpm)}

Se puede apreciar como a nivel celular hay un ensanchamiento del citoplasma, de la pared celular; de la célula en general en la muestra clinorotada. 
Figura 9. Control 1g. (Corte longitudinal 400 veces).

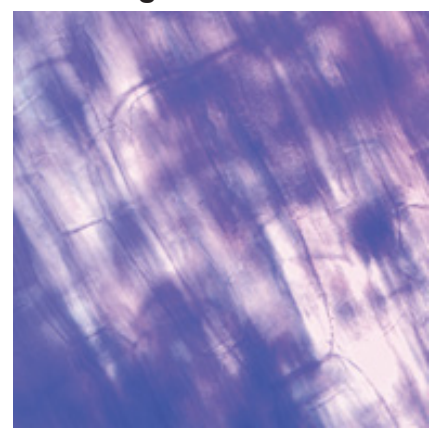

Fuente: Medina, Brenda.2017. Análisis gravitrópico de la raíz primordial de plantas mesoamericanas, en condiciones de microgravedad simulada

Figura 10. Clinorotada (Corte longitudinal 400 veces).

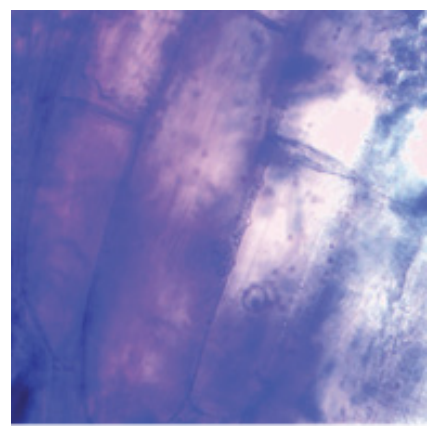

Fuente: Medina, Brenda.2017. Análisis gravitrópico de la raíz primordial de plantas mesoamericanas, en condiciones de microgravedad simulada

Gráfico 7. Muestra la longitud de crecimiento en centímetros vrs. El tiempo de germinación, para Phaseolus lunatus. Muestra Control 1g (Izquierda) y la muestra Clinorotada (Derecha).

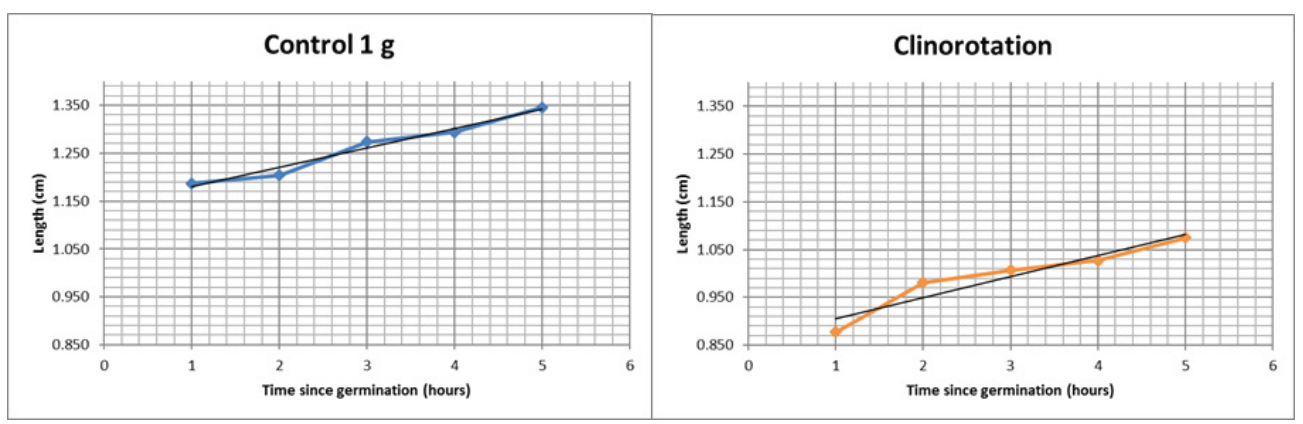

Fuente: Medina, Brenda.2017. Análisis gravitrópico de la raíz primordial de plantas mesoamericanas, en condiciones de microgravedad simulada 
Corte Histológico Phaseolus lunatus (70 rpm)

Figura 11. Control 1g. (Corte longitudinal a 100 veces).

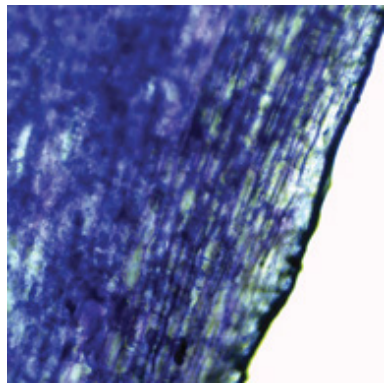

Fuente: Medina, Brenda.2017. Análisis gravitrópico de la raíz primordial de plantas mesoamericanas, en condiciones de microgravedad simulada

Figura 12. Clinorotada (Corte longitudinal a 100 veces).

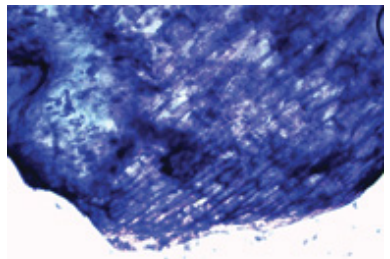

Fuente: Medina, Brenda.2017. Análisis gravitrópico de la raíz primordial de plantas mesoamericanas, en condiciones de microgravedad simulada

Gráfico 8. Izquierda, tasa angular promedio; La muestra Girada $90^{\circ}$ presento una curvatura de $4.179^{\circ} / \mathrm{h}$ y la muestra Clinorotada $0.645^{\circ} / \mathrm{h}$. Derecha, tasa de crecimiento promedio; la muestra Control $1 \mathrm{~g}$. creció $0.201 \mathrm{~cm}$ en una hora mientras que la muestra Clinorotada sometida a microgravedad creció $0.232 \mathrm{~cm}$ en ese mismo tiempo.

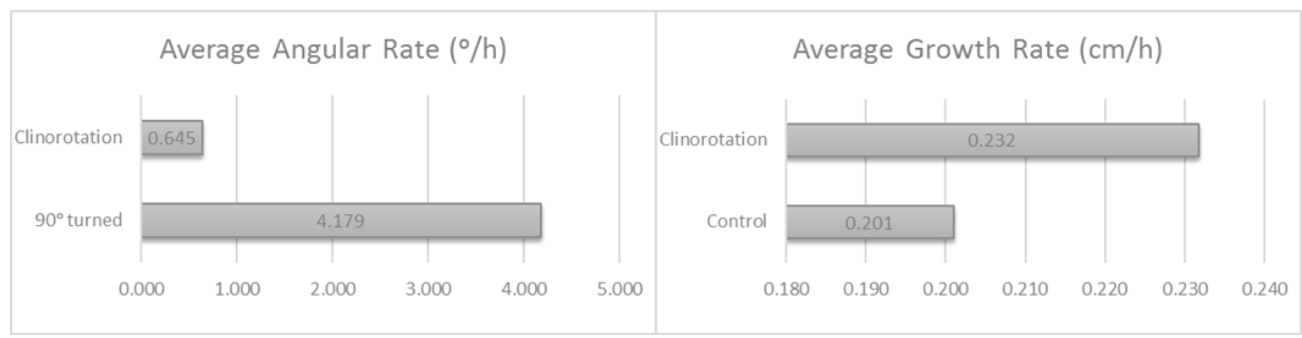

Fuente: Medina, Brenda.2017. Análisis gravitrópico de la raíz primordial de plantas mesoamericanas, en condiciones de microgravedad simulada 
Gráfico 9. Muestra la longitud de crecimiento en centímetros vrs. El tiempo de germinación, para Vigna unguiculata "Caupí gorda". Muestra Control Ig (Izquierda) y la muestra Clinorotada (Derecha).

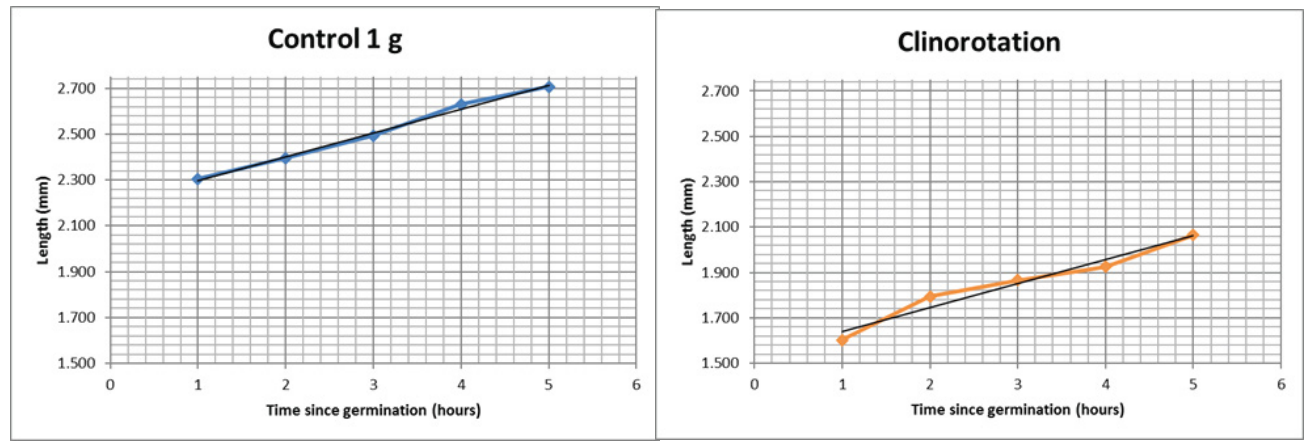

Fuente: Medina, Brenda.2017. Análisis gravitrópico de la raíz primordial de plantas mesoamericanas, en condiciones de microgravedad simulada

\section{Corte Histológico Vigna unguiculata (70rpm)}

Figura 13 Control 1g. (Corte longitudinal a 100 veces).

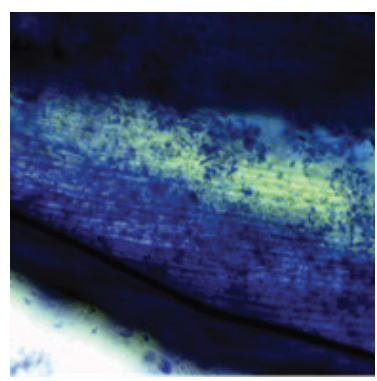

Fuente: Medina, Brenda.2017. Análisis gravitrópico de la raíz primordial de plantas mesoamericanas, en condiciones de microgravedad simulada

Figura 14 Clinorotada (Corte longitudinal a 100 veces).

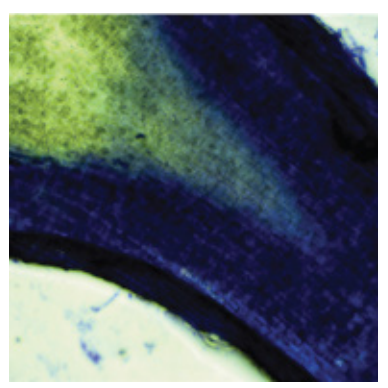

Fuente: Medina, Brenda.2017. Análisis gravitrópico de la raíz primordial de plantas mesoamericanas, en condiciones de microgravedad simulada 


\section{Sorghum bicolor var Sureño (70rpm-II)}

Gráfico 10. Izquierda, Tasa angular promedio; Los resultados arrojados por la muestra Girada $90^{\circ}$ mostraron que el ángulo se cerraba en una tasa $6.224 \%$ mientras que la muestra Clinorotada abría su ángulo 1.637 $\% / \mathrm{h}$. Derecha, Tasa de crecimiento promedio; la muestra Clinorotada creció el doble que la muestra Control 1g. La muestra Control creció $0.015 \mathrm{~cm}$ en una hora.
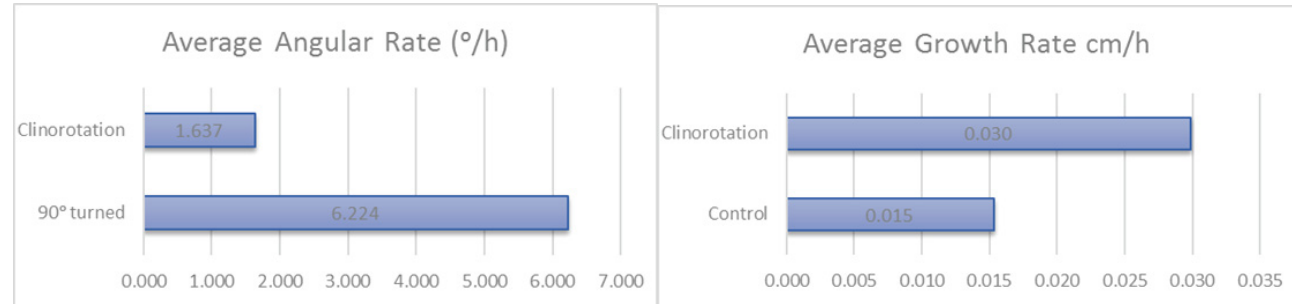

Fuente: Medina, Brenda.2017. Análisis gravitrópico de la raíz primordial de plantas mesoamericanas, en condiciones de microgravedad simulada

Gráfico 11. Muestra la longitud de crecimiento en centímetros vrs. El tiempo de germinación, para Sorghum bicolor var Sureño. Muestra Control $1 \mathrm{~g}$ (Izquierda) y la muestra Clinorotada (Derecha).

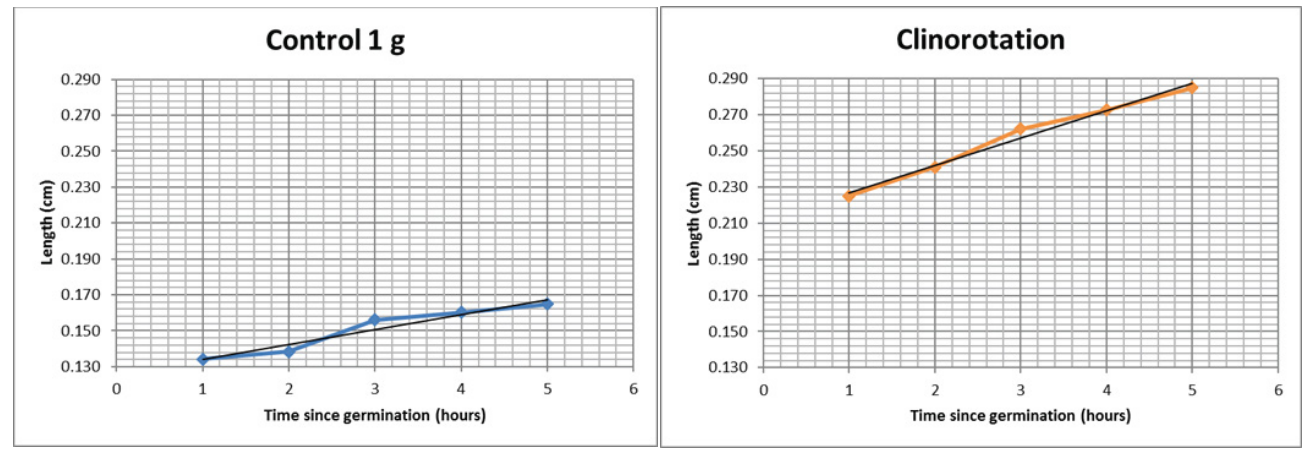

Fuente: Medina, Brenda.2017. Análisis gravitrópico de la raíz primordial de plantas mesoamericanas, en condiciones de microgravedad simulada 


\section{Corte Histológico Sorghum bicolor (70rpm-II)}

Figura 15. Control 1g. (Corte transversal a 400 veces).

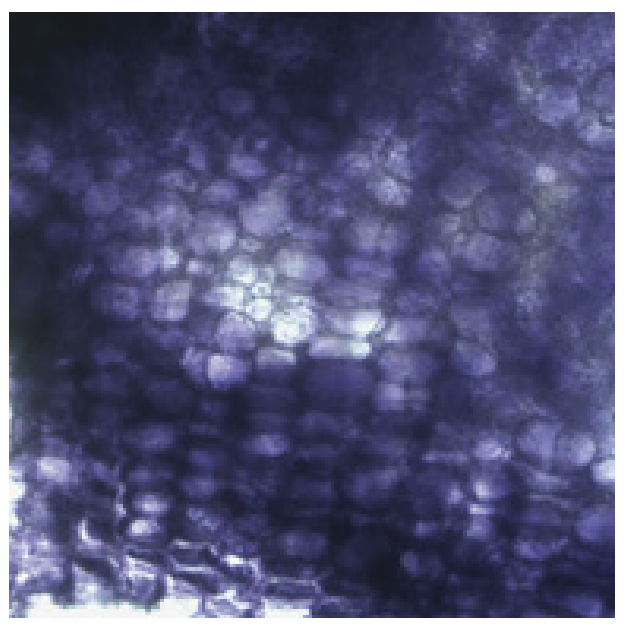

Fuente: Medina, Brenda.2017. Análisis gravitrópico de la raíz primordial de plantas mesoamericanas, en condiciones de microgravedad simulada

\section{Figura 16. Clinorotada (Corte transversal a 400 veces).}

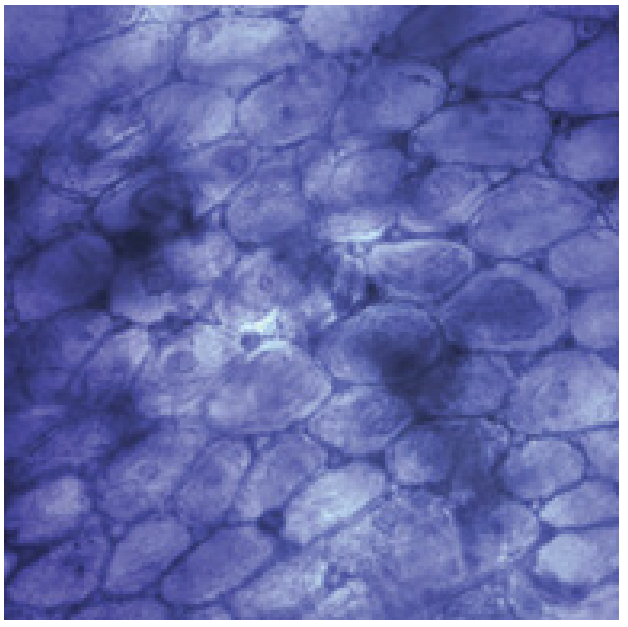

Fuente: Medina, Brenda.2017. Análisis gravitrópico de la raíz primordial de plantas mesoamericanas, en condiciones de microgravedad simulada 
Tabla 1. Resultados para las tres pruebas de clinorotación, dos de ellas a 70 rpm y otra a $90 \mathrm{rpm}$. Paras las seis especies que involucra el estudio. (A.A.R.= Tasa angular promedio y A.G.R.= Tasa de crecimiento promedio)

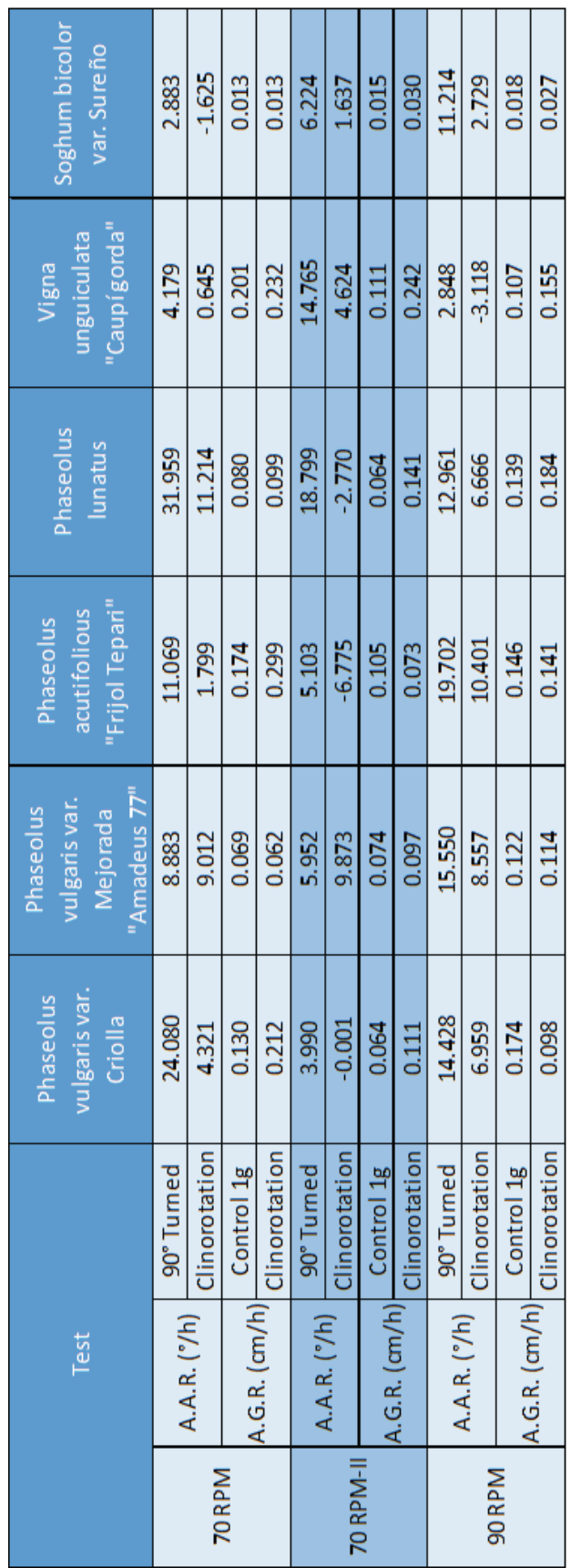




\section{Discusión de los resultados}

\section{Phaseolus vulgaris var. Criolla}

Se pudo observar que para Phaseolus vulgaris var. Criolla. Las tres pruebas, dos de ellas a velocidad de $70 \mathrm{rpm}$ y una a $90 \mathrm{rpm}$, la muestra Girada $90^{\circ}$ es decir la muestra que se mantuvo a gravedad natural $9.81 \mathrm{~m} / \mathrm{s} 2$ pero se giró $90^{\circ}$ para observar la curvatura se vio superada por la muestra Clinorotada. Respecto a la tasa de crecimiento en ambas pruebas a 70 rpm la muestra Clinorotada (Sometida a microgravedad) superó en crecimiento a la muestra Control 1g. (Gravedad natural $9.8 \mathrm{~m} / \mathrm{s} 2$ ) podría decirse que logró estimularla. Excepto en la prueba a velocidad de $90 \mathrm{rpm}$, en donde se podría suponer el estrés a la que fueron sometidas las células de crecimiento de la muestra Clinorotada, inhibieron el crecimiento; siendo así superada por la muestra Control 1g.

\section{Phaseolus vulgaris var. "Amadeus 77"}

En ambas pruebas a 70 rpm se pudo observar que la muestra Girada $90^{\circ}$ cerro más su ángulo de curvatura gravitrópica que la muestra clinorotada la cual en ambas mostro valores de $9^{\circ}$ en una hora.

En la prueba a $90 \mathrm{rpm}$ arrojo un resultado diferente en donde, la muestra clinorotada cerro más su ángulo en relación a la muestra Girada $90^{\circ}$.

Con respecto a la tasa de crecimiento los resultados fueron variados, para la primera prueba a $70 \mathrm{rpm}$ y la prueba a $90 \mathrm{rpm}$ a muestra Control (a gravedad natural 9.81 $\mathrm{m} / \mathrm{s} 2$ ) supero por poco a la muestra Clinorotada. Exceptuando la segunda prueba de 70 rpm (70RPM-II) en la cual la muestra clinorotada superó a la muestra Control por solo $0.023 \mathrm{~cm}$.

\section{Phaseolus acutifolious}

Con respecto a la tasa angular promedio para Phaseolus acutifolious, las tres pruebas arrojaron que la muestra Clinorotada presentó en promedio un menor ángulo que la muestra Girada $90^{\circ}$. Es decir, su ángulo de curvatura se orientó eficientemente en función del vector gravedad.

Respecto a la tasa de crecimiento, la muestra clinorotada en la prueba a $70 \mathrm{rpm}$ supero en crecimiento a la muestra Control $1 \mathrm{~g}$ por $0.055 \mathrm{~cm}$. La segunda prueba que 
involucra esta misma velocidad de rotación presento datos contrarios a la primera, en este caso la muestra control superó a la muestra Clinorotada, pero solamente por $0.032 \mathrm{~cm}$. Sorprendentemente la prueba a 90 rpm arrojó resultados similares tanto para la muestra Clinorotada $(0.141 \mathrm{~cm} / \mathrm{h})$ como la muestra Control $1 \mathrm{~g} .(0.146 \mathrm{~cm} / \mathrm{h})$.

\section{Phaseolus lunatus}

Para Phaseolus lunatus se pudo ver que en las tres pruebas la muestra Clinorotada sustento menores ángulos que la muestra Girada $90^{\circ}$. Respecto a la tasa de crecimiento se obtuvieron similares resultados en las tres pruebas realizadas, es decir que la muestra Clinorotada superó en crecimiento a la muestra Control 1g. En la primera (70 rpm) por $0.019 \mathrm{~cm}$, en la segunda $(70 \mathrm{rpm}-\mathrm{II})$ por $0.077 \mathrm{~cm}$ y en la última (90 rpm) por $0.045 \mathrm{~cm}$.

Para Vigna unguiculata las tres pruebas realizadas, arrojaron que la muestra Girada $90^{\circ}$ presentó un ángulo mayor que la muestra Clinorotada. Esto igualando a la especie anterior, tanto en la tasa angular como a la tasa de crecimiento. En la primera (70 rpm) la muestra Clinorotada supera a su par Control $1 \mathrm{~g}$. por $0.031 \mathrm{~cm}$, en la segunda (70 rpm-II) por $0.131 \mathrm{~cm}$ y en la última $(90 \mathrm{rpm})$ por $0.048 \mathrm{~cm}$.

\section{Sorghum bicolor var Sureño}

Para Esta especie se observó que la muestra Girada $90^{\circ}$ presento un ángulo mayor que la muestra Clinorotada en las tres pruebas coincidiendo con las dos especies anteriores.

La Tasa de crecimiento fue muy pareja entre la muestra Clinorotada y la muestra Control $1 \mathrm{~g}$. de la primera prueba a $70 \mathrm{rpm}$, obteniendo porcentajes iguales. Mientras que para la segunda a 70 rpm-II, como también a 90 rpm. La muestra Clinorotada superó en crecimiento a la muestra Control $1 \mathrm{~g}$.

\section{CONCLUSIONES}

1. Phaseolus vulgaris var. Criolla y variedad Amadeus 77 parecen ser especies prometedoras para continuar haciendo pruebas en Tierra con microgravedades entre $8.22 \times 10-2 \mathrm{~m} / \mathrm{s} 2$ a $1.359 \times 10-1 \mathrm{~m} / \mathrm{s} 2$; Ya que el crecimiento fue estimulado para la primera y no fue considerablemente afectado para la segunda. Pero es 
preciso seguir investigando estas especies que son de vital importancia para la región Mesoamericana, especialmente Honduras que es donde se creó la especie mejorada Amadeus 77.

2. Phaseolus acutifolious presento un excelente desempeño en cuanto a crecimiento en microgravedad 1.359x10-1 m/s2. Donde la variación entre la muestra Clinorotada y la muestra Control con respecto a la tasa de crecimiento fue de $0.005 \mathrm{~cm}$. El resultado a microgravedad $8.22 \times 10-2 \mathrm{~m} / \mathrm{s} 2$ (provocado por una velocidad de rotación a 70 rpm) aún es incierto. Ya que dos pruebas fueron realizadas y mostraron resultados contradictorios, la primera reveló que la muestra Clinorotada sobrepasaba en longitud a la muestra Control $1 \mathrm{~g}$. por $0.125 \mathrm{~cm}$. Mientras que la segunda prueba reveló que la muestra Control $1 \mathrm{~g}$. supera a la muestra Clinorotada por 0.032 $\mathrm{cm}$.

3. Phaseolus lunatus y Vigna unguiculata son especies que se ve estimuladas por la microgravedad, o que se adaptan muy bien a ella. De las tres pruebas que se realizaron a microgravedades de $8.22 \times 10-2 \mathrm{~m} / \mathrm{s} 2$ y $1.359 \times 10-1 \mathrm{~m} / \mathrm{s} 2$, muestran un crecimiento óptimo para este tipo de frijol. $Y$ en el caso de Vigna unguiculata al no mostrar enormes variaciones con respecto a la tasa angular promedio el ápice de la raíz primordial se puede decir que no se ve fuertemente afectada por la microgravedad.

4. La raíz primordial de Sorghum bicolor mostro una buena adaptación al proceso de microgravedad. La muestra Clinorotada igualó y/o superó en longitud a la muestra Control $1 \mathrm{~g}$. Y por otra parte el comportamiento de la tasa angular promedio no varió significativamente. Fue una de las especies que menos variaciones tuvo en las sesiones de microgravedad.

5. Phaseolus lunatus, Vigna unguiculata y Soghum bicolor son tres especies de importancia en la agricultura actual Mesoamericana (y no solamente esta área en la cual se enfoca esta investigación sino también para toda Centroamérica y el Caribe) se pudo observar que su crecimiento no ha sido considerablemente afectado a baja gravedad, los resultados entre muestra. Por lo que son especies que podrían ser consideradas para mayores estudios en microgravedad a bordo de sondas espaciales.

6. Los resultados arrojados por la investigación muestran que efectivamente la muestra "Clinorotada" presentó alteraciones respecto a la curvatura gravitrópica presentada por muestra "Girada 90" para las 6 especies estudiadas. También se pudo ver 
que exhibieron alteraciones en el patrón de crecimiento entre la muestra "Control 1g." y la muestra "Clinorotada". Así como también las variaciones en el tejido estructural. Por lo que se aprueban las hipótesis realizadas en el planteamiento del problema en la investigación.

\section{RECOMENDACIONES}

1. Se recomienda que la investigación continúe, para seguir ampliando el conocimiento no solamente con las especies trabajadas en esta investigación, si no también incorporando nuevas especies para observar su comportamiento frente a microgravedad.

2. Ya que esta ha sido una investigación pionera en el área Mesoamericana se recomienda, investigar que sucede con los brotes de las especies estudiadas en este trabajo.

3. Cuando se planteó la investigación se definió trabajar con Calabaza criolla (Cucurbita maxima), esta especie presenta algunos inconvenientes ya que la especie necesita un tiempo mayor a una semana para su germinación. Por lo que algunas clases de hongos comienzan a atacar la semilla sin germinar y el lote de investigación (Cuatro muestras en los discos de Petri) se pierde, y debe descartarse. Por lo que no se recomienda trabajar con esta especie para futuras investigaciones de este tiempo.

\section{BIBLIOGRAFÍA}

Braun, M., \& Limbach, C. (2006). Rhizoids and protonemata of characean algae model cells for research on polarized growth and plant gravity sensing. Protoplasma, 229, volumen (número), pp. 133-142.

Herranz, R., Anken, R., Boonstra, J., Braun, M., Christianen, P. C., de Geest, M., . . . Hilbig, R. (2013). Ground-Based Facilities for Simulation of Microgravity: Organism-Specific Recommendations for Their Use, and Recommended Terminology. ASTROBIOLOGY, 13(1), páginas.

Office for Outer Space Affairs United Nations Office at Vienna. (2013). Teacher's Guide Plant Experiments in Microgravity. New York: United Nations. 